Elsevier required licence: (C) <2018>. This manuscript version is made available under the CC-BY-NC-ND 4.0 license http://creativecommons.org/licenses/by-nc-nd/4.0/ 
Journal of Geriatric Oncology. https://doi.org/10.1016/j.jgo.2018.07.004

\section{Utilisation of geriatric assessment in oncology - a survey of Australian medical oncologists}

Timothy H. M. To ${ }^{a, b}$, Wee Kheng Soo ${ }^{c, d, e}$, Heather Lane ${ }^{f}$, Adnan Khattak ${ }^{g, h}$, Christopher Steer ${ }^{i, j}$, Bianca Devitt ${ }^{d, k}$, Haryana M. Dhillon', Anne Booms ${ }^{m, n, o, p}$, Jane Phillips ${ }^{q}$

\section{Affiliations}

a. Division Rehabilitation, Aged Care and Palliative Care, Flinders Medical Centre, Bedford Park, South Australia, Australia.

b. College of Nursing and Health Sciences, Flinders University, Bedford Park, South Australia, Australia

c. Eastern Health Clinical School, Monash University, Victoria, Australia. E-mail: kheng.soo@monash.edu

d. Department of Medical Oncology, Eastern Health, Victoria, Australia

e. Department of Geriatric Medicine, Eastern Health, Victoria, Australia

f. Rockingham General Hospital, Rockingham, Western Australia, Australia. E-mail: Heather.Lane@health.wa.gov.au

g. Department of Medical Oncology, Fiona Stanley Hospital, Murdoch, Western Australia, Australia. Email:adnan.khattak@health.wa.gov.au

h. Murdoch University, Perth, Western Australia, Australia

i. Border Medical Oncology, Albury, New South Wales, Australia. E-mail: christopher.steer@bordermedonc.com.au

j. Rural Clinical School, University of New South Wales, Albury, New South Wales, Australia

k. Eastern Health Clinical School, Monash University, Victoria, Australia. E-mail: bianca.devitt@monash.edu

I. Centre for Medical Psychology and Evidence-based Decision-making (CeMPED), School of Psychology, University of Sydney, Sydney, New South Wales, Australia. E-mail: haryana.dhillon@sydney.edu.au

m. Capital Regional Cancer Centre, ACT Health, Garran, Australian Capital Territory, Australia. E-mail: anne_booms@hotmail.com

n. John James Medical Centre, Deakin, Australian Capital Territory, Australia

o. National Capital Private Hospital, Garran, Australian Capital Territory, Australia

p. School of Nursing, The University of Canberra, Canberra, Australian Capital Territory, Australia

q. Improving Palliative, Aged and Chronic Care through Clinical Research and Translation (IMPACCT), Faculty of Health, University of Technology Sydney, Sydney, New South Wales, Australia. E-mail: Jane.Phillips@uts.edu.au

\section{Corresponding author:}

Dr Timothy To, Division of Rehabilitation, Aged Care \& Palliative Care, Rehabilitation and Palliative Care Building, 4th Floor, Flinders Medical Centre, Flinders Drive, Bedford Park SA 5042. Phone: +61-8-84042660, Fax: +61-8-84042249,

E-mail: Timothy.To@sa.gov.au

Keywords: medical oncology, geriatric assessment, frailty, survey 


\begin{abstract}
Introduction

Geriatric assessment (GA) is a multidimensional health assessment of the older person to evaluate their physical and cognitive function, comorbidities, nutrition, medications, psychological state, and social supports. GA may help oncologists optimise care for older patients with cancer. The aim of this study was to explore the views of Australian medical oncologists regarding the incorporation of geriatric screening tools, GA and collaboration with geriatricians into routine clinical practice.
\end{abstract}

\title{
Methods
}

Members of the Medical Oncology Group of Australia were invited to complete an online survey that evaluated respondent demographics, practice characteristics, treatment decision-making factors, use of GA, and access to geriatricians.

Results

Sixty-nine respondents identified comorbidities, polypharmacy, and poor functional status as the most frequent challenges in caring for older patients with cancer. Physical function, social supports and nutrition were the most frequent factors influencing treatment decision-making. The majority of respondents perceived value in GA and geriatrician review, although access was a barrier for referral. Such services would need to be responsive, providing reports within two weeks for the majority of respondents.

\section{Conclusion}

Despite an emerging evidence base for the potential benefits of GA and collaboration with geriatricians, medical oncologists reported a lack of access but a desire to engage with these services. 


\section{Introduction}

A growing number of older adults in Australia are being diagnosed with cancer. This is due to a combination of an ageing population and increased cancer incidence with age. A detailed assessment of the older person's health status can guide the appraisal of risks and benefits of cancer treatment, influence treatment choice and intensity and guide supportive care interventions.

Assessment of physical and cognitive function, comorbid medical conditions, nutrition, medication usage, psychological state ${ }_{2}$ and social supports comprise the core domains of geriatric assessment (GA).

Internationally, an increasing number of cancer services have adopted systematic approaches to the care of older adults with cancer by incorporating GA and geriatric expertise into assessment and decision-making processes ${ }^{1}$. In comparison, GA and geriatric oncology services remain confined to a small number of cancer centres in Australia ${ }^{1}$.

Australia has a universal healthcare system for permanent residents covering medical consultations, public hospital admissions and subsidised pharmaceuticals. In 2016, there were 619 geriatricians in Australia with an average of 2.4 clinicians per 100,000 population ${ }^{2}$. Geriatric medicine and cancer services, although readily available within both public and private hospitals, often lack a formalised approach to collaboration. To date, there is limited information on Australian oncologists' views and experiences of geriatric oncology.

This study aimed to explore the views of Australian medical oncologists regarding the perception of, and barriers to the incorporation of geriatric screening tools, GA and collaboration with geriatricians in routine clinical practice.

\section{Methods}

A cross-sectional survey was developed by a multidisciplinary national expert steering group on behalf of the Clinical Oncology Society of Australia's Geriatric Oncology group, comprising medical and nursing representatives from medical oncology, geriatric medicine, and palliative care. The online survey, based on a literature review and expert opinion, comprised 30 questions covering: (i) respondent characteristics, clinical practice environment and patient population; (ii) challenges and treatment decision-making factors in the management of older patients with cancer; and (iii) benefits of and barriers to the implementation of GA or geriatrician review in cancer care for older patients.

For the purpose of this study, geriatric screening tools were defined as brief tools used to identify older patients with cancer who are likely to benefit from a more detailed assessment. Geriatric assessment was defined as a panel of assessment tools covering relevant domains for the older patient with cancer, which identify domains of concern potentially requiring further assessment and intervention. This was distinct from a geriatrician review, in which a geriatrician assesses the older person, contextualises information to the patient's situation, and makes recommendations to guide interventions. These definitions were presented to respondents in relevant sections of the survey.

An invitation to participate in the online survey was emailed to members of the Medical Oncology Group of Australia (MOGA) in June 2015, comprising 450 oncologists and 172 trainees, representing approximately $90 \%$ of medical oncologists in the region. Two reminders were sent before the survey closed in November 2015 to encourage participation. 
The survey was completed by participants using SurveyMonkey (SurveyMonkey Inc., San Mateo, California, USA). Descriptive statistical analysis was performed using SPSS version 24 (IBM Corp., Armonk, NY). A coding schema was developed to categorise responses to open-ended questions into broad themes. Group comparisons of respondent characteristics were performed for the following three questions: 'Do you or your service routinely screen your older cancer patients?'; 'Have you ever referred a patient for geriatric assessment?'; and, 'Have you ever referred a patient for a geriatrician review?'.

This study was approved by the University of Technology Sydney's Human Research Ethics Committee (05 May 2015; Approval Number: 2015000122).

\section{Results}

Sixty-nine oncologists completed the survey (response rate 11\%). Table 1 outlines respondent characteristics. Respondents reported older patients constituted a substantial portion of their practice, with $33 \%$ (23/69) estimating $51-75 \%$ of their patients were over 70 years, and a further $54 \%$ estimating $25-50 \%$ of patients were over 70 years. Fifty-nine percent of respondents reported an interest in geriatric oncology. Allied health services were 'readily' or 'somewhat' available for over $95 \%$. No respondent characteristics (years of practice, practice type, location) were associated with a higher likelihood of conducting geriatric screening, or referring for GA or geriatrician review.

\section{Decision-making for older patients}

Comorbidities, polypharmacy, poor functional status and treatment toxicity were the most frequently identified challenges when caring for older patients with cancer (Figure 1). Physical function, living circumstance/social supports, and nutritional status were most frequently identified as important treatment decision factors for older patients with cancer (Figure 2).

\section{Geriatric screening}

Only $19 \%(13 / 69)$ of respondents used geriatric screening tools in routine practice. Of these respondents, screening was primarily triggered by age (77\%), most commonly an age of 70 years (80\%). The remainder screened any patient they suspected to be frail. Screening tools used are outlined in Figure 3, with the Mini-Mental State Examination most commonly used.

\section{Geriatric assessment}

The majority of respondents identified that GA added to clinical assessment (71\%) and influenced clinical decision-making (65\%) (Figure 4). GA had been requested by $56 \%$ of respondents. Access to $(40 \%)$ and timeliness of GA (47\%) were identified as barriers for the 30 respondents who had not requested $G A$, whilst $23 \%$ of these saw no need for $G A$.

\section{Geriatrician review}

Access to timely geriatrician review was desired by $74 \%$ (52/69) of respondents. However, only $30 \%$ had referred to a geriatrician, of whom $90 \%$ had referred less than 10 patients in the last year. Geriatrician review was identified as adding to clinical assessment (73\%) and influencing clinical assessment (66\%) (Figure 4). 
Issues triggering referral to a geriatrician included concerns regarding: cognition and decisionmaking capacity 51\% (23/45), general health and frailty (24\%), assessment and management of comorbidities (24\%), functional assessment and optimisation (22\%) and medication review (16\%).

If available, $57 \%$ (39/68) of respondents estimated referring less than $25 \%$ of their patients aged over 70 to a geriatrician, with $25 \%$ of respondents estimating referring $25-50 \%$. The remainder $(17 \%)$ estimated referring more than half of their older patients. Referrals would generally be at diagnosis $(28 \%)$ or prior to a treatment decision (62\%).

Importantly, a report from a GA or geriatrician would ideally be finalised within one week for $60 \%$ $(41 / 68)$ of respondents, or two weeks for $37 \%$. A written report was preferred by the majority of respondents $(78 \%)$, although a verbal report $(26 \%)$ or as part of a multidisciplinary discussion (56\%) were acceptable for many.

\section{Barriers}

Identified barriers to GA or geriatrician review are presented in Figure 5. The top three identified barriers to GA or geriatrician review were: time required to perform GA $(75 \%)$, lack of geriatrician (63\%), and lack of services available to perform GA (62\%) (Figure 5).

\section{Discussion}

This study identified a perception that GA and/or geriatrician review would improve clinical assessment and influence decision-making amongst participating medical oncologists. These findings are similar to other international studies where the vast majority (75-95\%) of cancer specialists believe GA is beneficial ${ }^{3-6}$.

In our study, comorbidities, polypharmacy, poor functional status and treatment toxicity were the most commonly identified challenges when caring for older patients with cancer, similar to those identified in a 2006 survey of Canadian cancer specialists ${ }^{7}$. However, polypharmacy ranked first in our survey, compared to eighth in Canada, perhaps reflecting increasing awareness of the risks of polypharmacy.

Patient factors that influenced treatment decisions included comorbidities, physical function and patient preferences, whereas treatment factors that most frequently influenced treatment decisions were benefits, toxicity, and prognosis, similar to those found in Canadian and Singaporean surveys ${ }^{5}$.

Factors predictive of chemotherapy toxicity in older patients, as identified in two scoring systems, include cognition, nutrition, falls, functional status and age, which are also key components of the $\mathrm{GA}^{89}$. Whilst physical function is now widely recognised by oncologists as an important GA domain, factors such as cognition, nutrition and falls appear less commonly recognised. This suggests ongoing education is required for routine incorporation of these domains into oncological assessment. However, in this survey, oncologists would most commonly consult a geriatrician about cognition, followed by comorbidities, general health, and physical function.

Access to geriatric expertise remains a major barrier for older adults with cancer in Australia, similar to surveys from other high-income countries ${ }^{45}$. In our study, despite the majority of respondents perceiving that GA improved clinical assessment and treatment decisions, $70 \%$ of respondents had 
never referred to a geriatrician. However, GA performed by allied health may help address this issue by identifying patients who would benefit from a detailed geriatrician review, thus potentially improving accessibility and reducing implementation costs. The majority of GA or geriatrician reviews in oncology were required within one week, and almost all within two, as these reviews are often required at time-critical periods, such as at diagnosis or time of treatment decisions to inform whether modified cancer treatment is required. However, GA may also inform supportive care interventions, which may not be as time-critical and can occur in parallel with oncological decisionmaking and treatment. In Belgium, 59\% of GA is performed the same day as the oncological assessment, with over $80 \%$ conducted within four days ${ }^{10}$. This difference likely reflects a systematic approach facilitating rapid GA and increased use of integrated inpatient oncological GA processes. The design of a GA or geriatrician consultation service for older patients with cancer must account for the need for quick response times.

Whilst the majority of respondents preferred the GA or geriatrician review report in writing, over half welcomed it as being part of a multidisciplinary discussion. Care planned by a multidisciplinary team facilitates collaboration and recognition of GA domains and higher rates of treatment modification $^{1112}$.

Concerns have been raised about the feasibility of geriatric oncology services without additional resources. A German survey found that whilst 95\% thought that GA was meaningful, $30 \%$ thought it impracticable ${ }^{6}$. Similarly in a Singaporean survey, $28 \%$ questioned the feasibility of such a service ${ }^{5}$. Feasibility issues could be addressed by examining alternative models of geriatric oncology, developing partnerships between existing oncology and geriatric services, using screening to identify vulnerable individuals for comprehensive assessment, and demonstrating the value of such services to stakeholders.

Even with a systematic approach to geriatric oncology such as in Belgium, the incorporation of GA and interventions into the care of older patients with cancer is challenging. A recent Belgian survey identified that whilst a substantial proportion of patients receive GA and have recommendations implemented, the availability and incorporation of GA into multidisciplinary discussions and treatment decisions occurs only less than $20 \%$ of the time ${ }^{10}$.

\section{Limitations}

Despite the low survey response rate, the findings are consistent with surveys conducted in other countries. Respondents likely had limited direct exposure to geriatric oncology services given the small number of services in Australia. Conversely, it is likely that there is a degree of responder bias, with those with an interest in geriatric oncology likely over-represented in the sample.

\section{Implications for future practice/service design}

This study has identified a desire and perceived need for increased geriatric oncology services and collaboration with geriatricians amongst respondents. However, the low response rate may also reflect an ambivalence towards geriatric oncology amongst oncologists, highlighting the need for ongoing awareness-raising and generation of supporting evidence. The design and provision of geriatric oncology services must address barriers identified in this research, in particular the availability of GA and geriatric expertise in a timely manner. Closer collaboration between 
oncologists and geriatric expertise is required to embed such services into routine practice. Given that geriatric medicine services and cancer care services are often co-located within Australian hospitals, utilisation of existing resources may be possible. Incorporation of geriatrics into oncology training, and vice versa, may build expertise into the future workforce, and should be considered by the respective training organisations. Dual training in geriatric medicine and oncology has been undertaken by a small number of clinicians in Australia. Whilst it will not be feasible for all older adults with cancer to see a dual-trained geriatric oncologist, these highly skilled specialists are ideally placed to guide future research and education in the field.

The Clinical Oncology Society of Australia is currently developing geriatric oncology guidelines to support clinicians and services to design and practice in accordance with current best evidence. Implementation of these guidelines could be supported by review of the Australian Medicare Benefits Schedule - GA is currently not reimbursed by Medicare but future reforms may support the incorporation of GA and other multidisciplinary care into oncological practice. The preferred model of care may be best addressed locally, tailored to the local availability of geriatric expertise and funding models. Ongoing research is required to build evidence regarding the merits of differing models of care and the benefits of GA-driven interventions in this population.

\section{Conclusion}

In this survey of medical oncologists, geriatric assessment of older patients with cancer and collaboration with geriatricians was welcomed. However, access to appropriate expertise was a substantial barrier. Furthermore, such services need to occur in a timely manner and work in close collaboration with oncologists in order to positively influence treatment decisions and outcomes. 
Table 1. Respondent characteristics $(n=69)$

\begin{tabular}{|l|l|c|}
\hline & & $\mathrm{n}^{*}(\%)$ \\
\hline Years of practice as specialist oncologist & In training & $14(20)$ \\
\hline & $0-10$ years & $27(39)$ \\
\hline & $11-20$ years & $12(17)$ \\
\hline & $21-30$ years & $11(16)$ \\
\hline & $30+$ years & $5(7)$ \\
\hline & & \\
\hline Practice type (majority) & Public & $41(59)$ \\
\hline & Private & $15(22)$ \\
\hline & Academic & $7(10)$ \\
\hline & Other & $6(9)$ \\
\hline & & \\
\hline & Full-time & $44(64)$ \\
\hline & Part-time & $25(36)$ \\
\hline Location & & $62(90)$ \\
\hline & Metropolitan & $6(9)$ \\
\hline & Regional & $1(1)$ \\
\hline
\end{tabular}

*Percentanges may not add up to $100 \%$ due to rounding 
Figure 1. Challenging factors in the management of older patients with cancer ( $n=69)$.

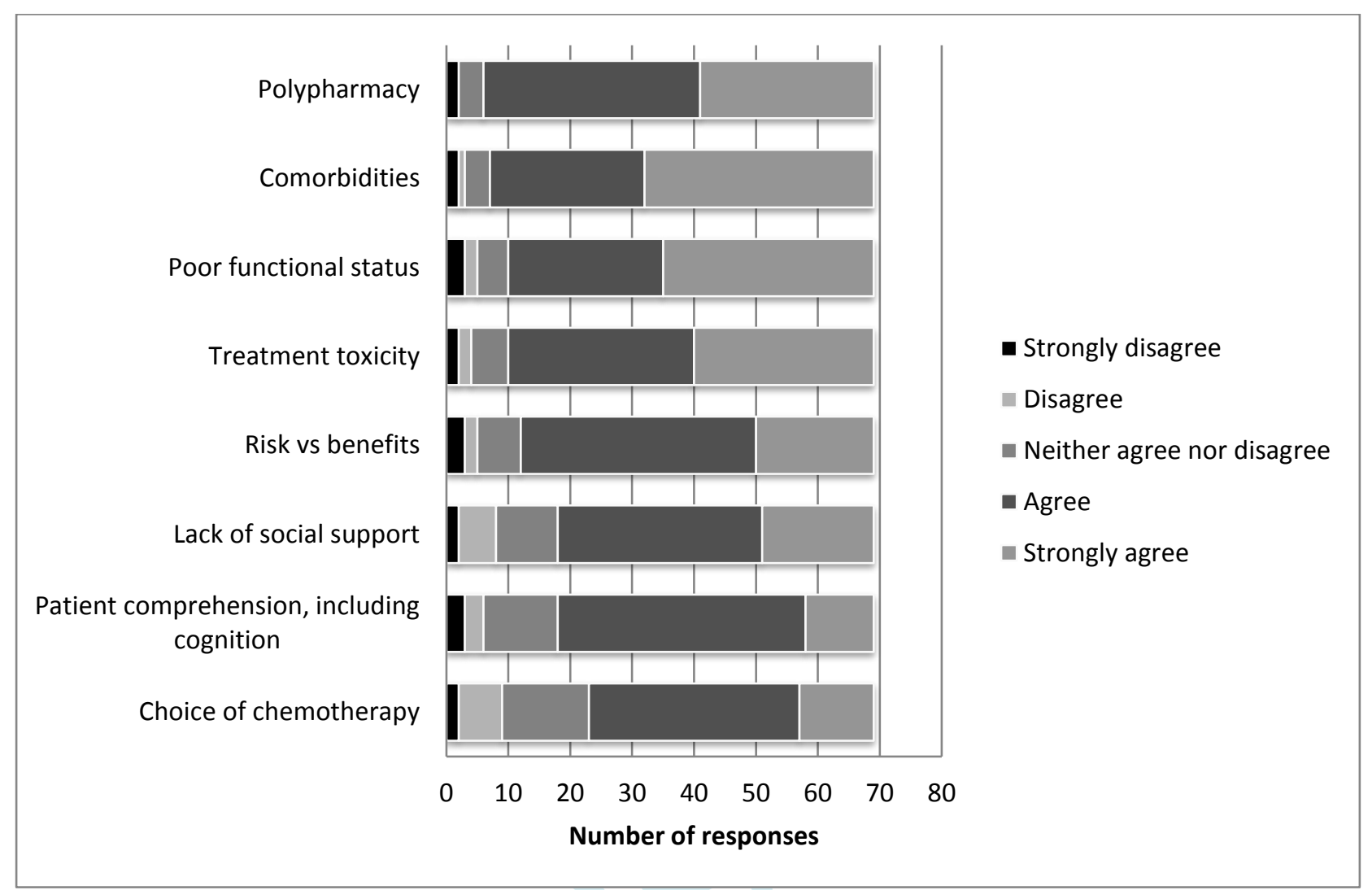


Figure 2. Factors considered important when making treatment decisions in patients with cancer aged 70 years and over $(n=69)$.

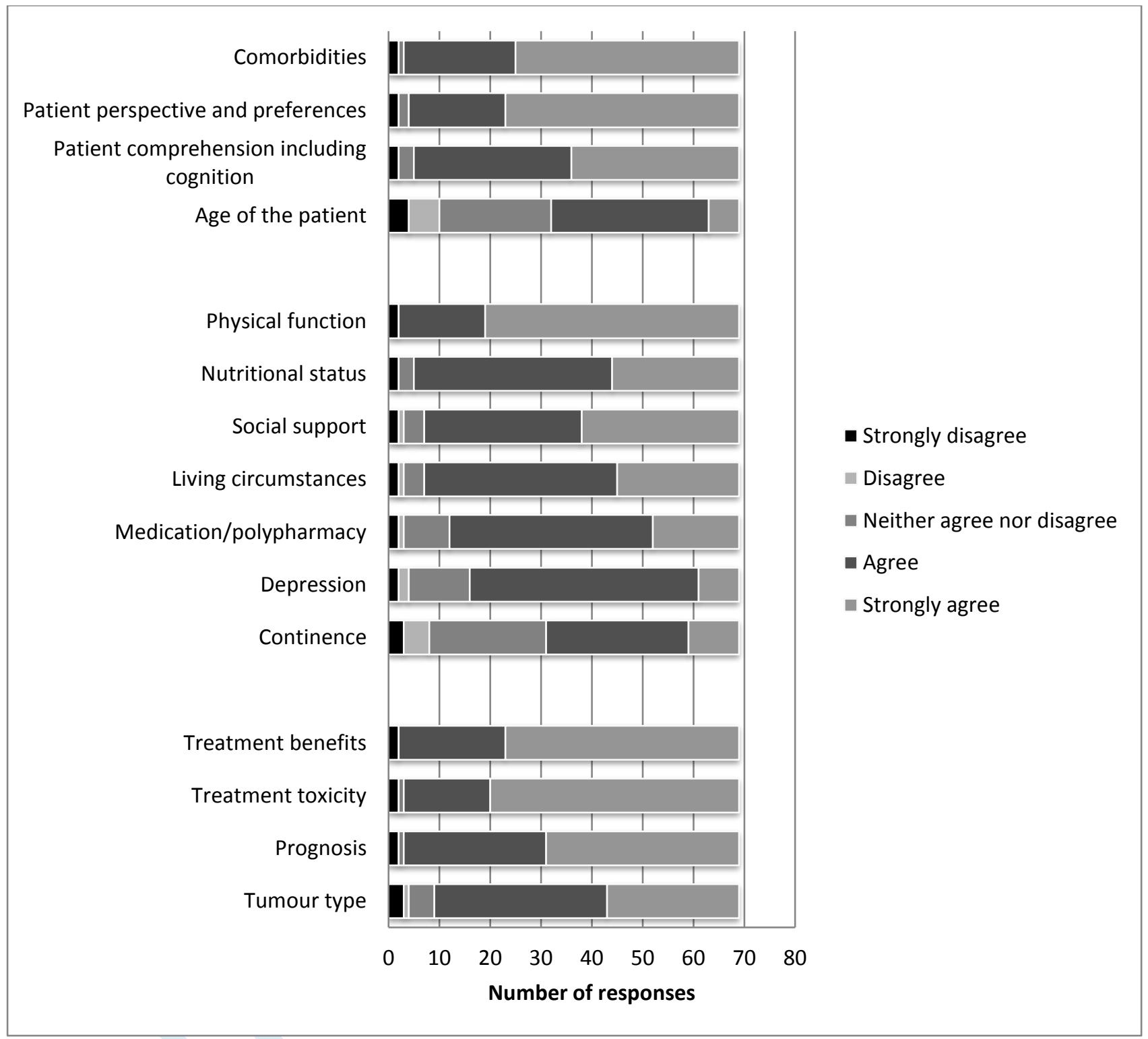


Figure 3. Currently used geriatric screening tools $(n=13)$.

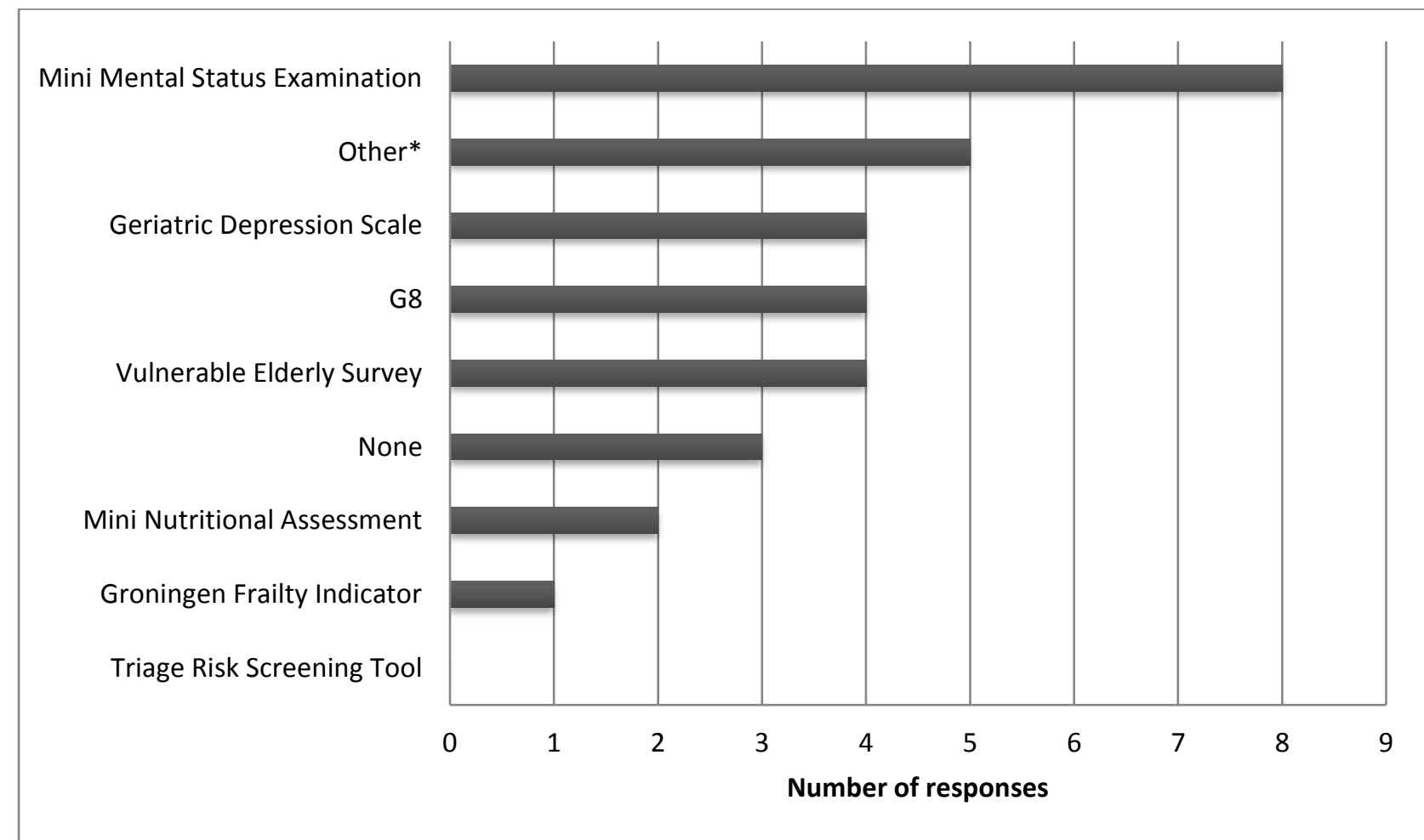

*Other includes - Instrumental and personal activities of daily living, timed up-and-go, Cancer and Aging Research Group chemotherapy toxicity tool, Montreal Cognitive Assessment, Charlson Comorbidity Index 
Figure 4. Impact of geriatric assessment and geriatrician review on medical oncologist's clinical assessment and decision-making $(n=69)$.

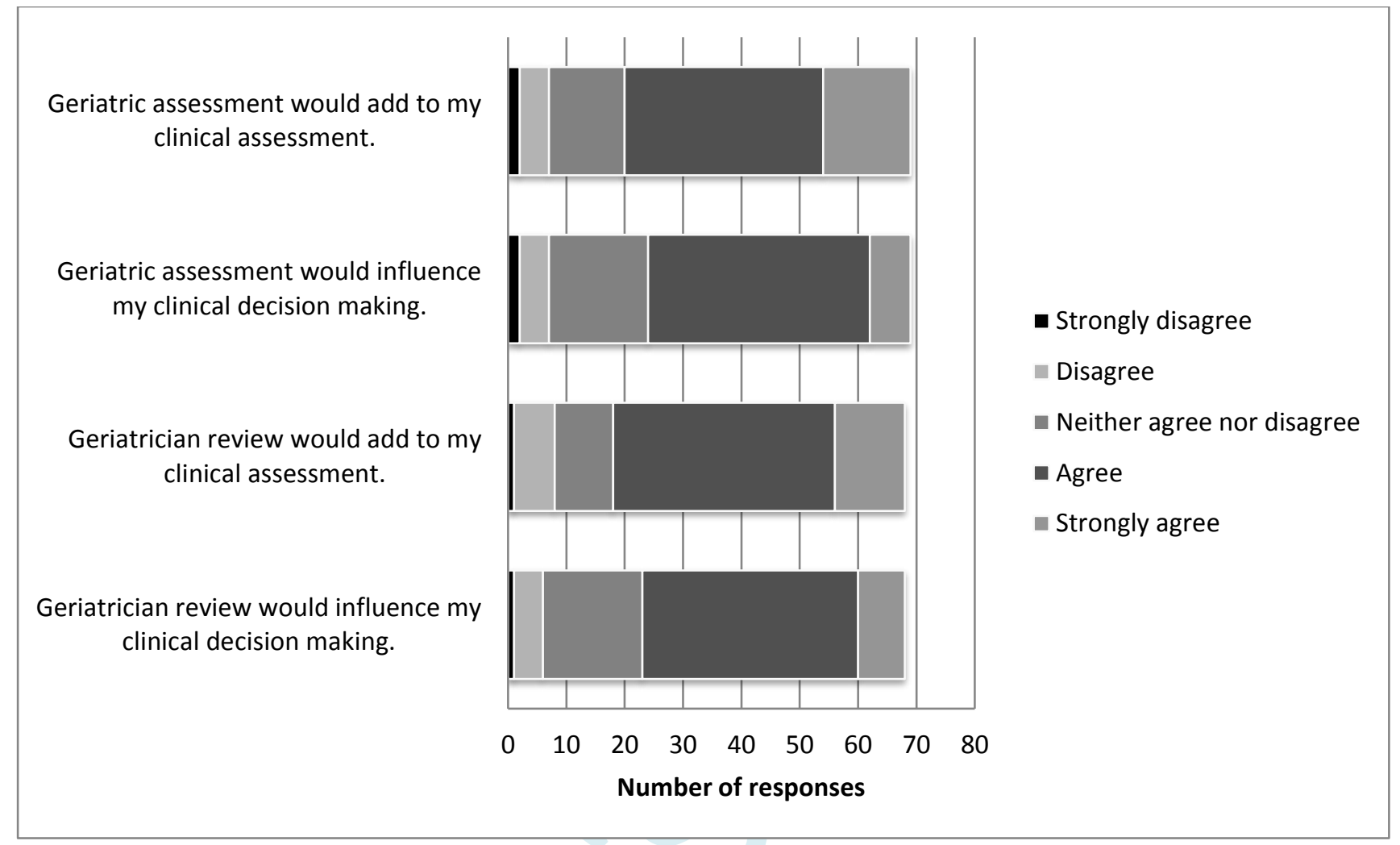


Figure 5. Barriers to implementation of geriatric assessment or geriatrician review of older patients with cancer $(n=65)$.

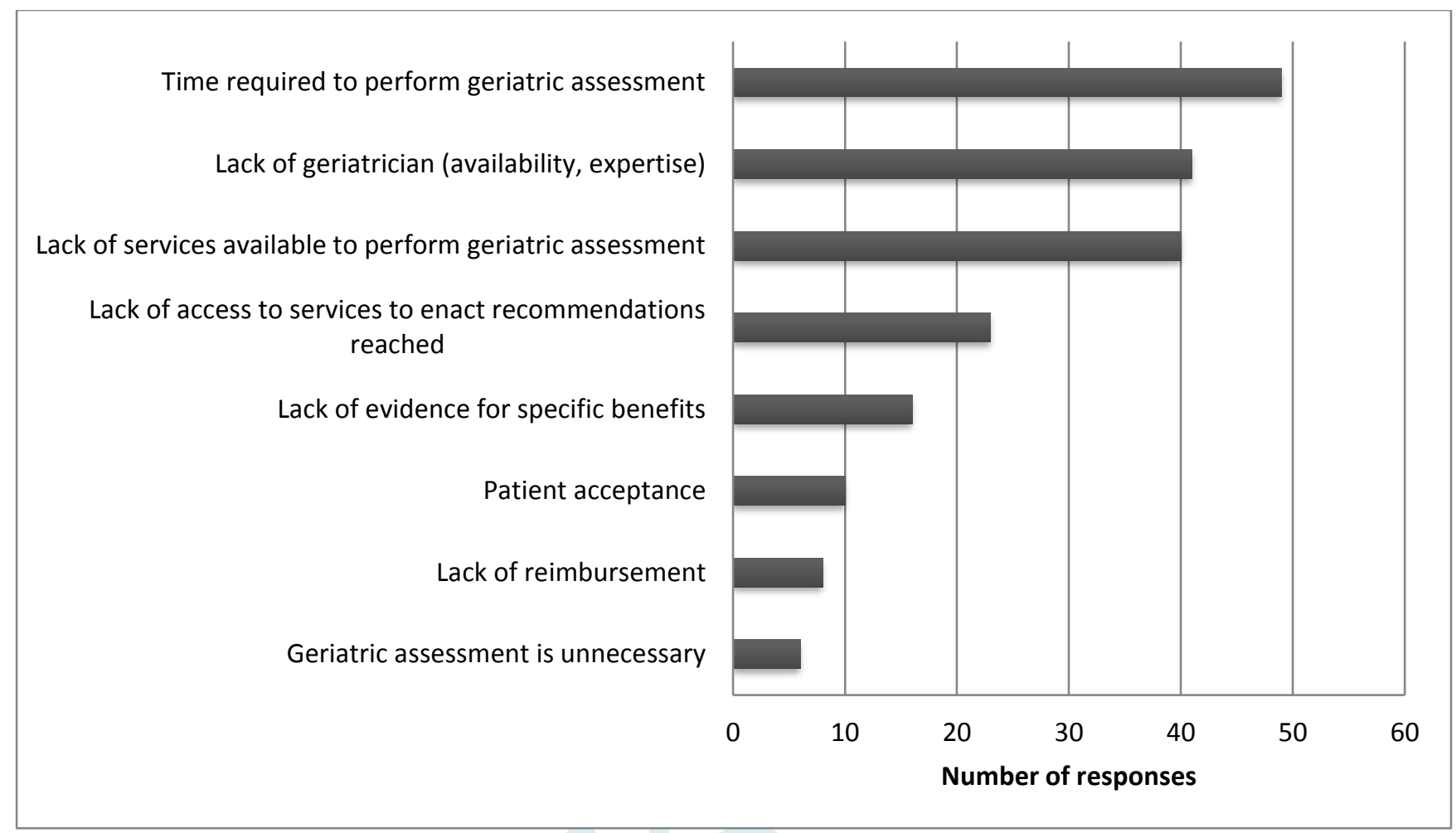




\section{Acknowledgements}

The authors would like to thank all those who participated in the survey, the Clinical Oncology Society of Australia (COSA) for their support and the guidance of the COSA Geriatric Oncology group.

Funding

The authors report no external sources of funding for this study.

\section{Conflict of interest}

C Steer reports personal fees from MSD, Roche and Janssen outside the submitted work, and is a member of the editorial board for the Journal of Geriatric Oncology. The other authors had no conflicts of interests to disclose.

\section{Author contributions}

Study Concepts: T To, W Soo, H Lane, A Khattak, B Devitt, C Steer, J Phillips Study Design: T To, W Soo, H Lane, A Khattak, B Devitt, C Steer, J Phillips Data Acquisition: T To, C Steer, J Phillips

Quality Control of Data and Algorithms: T To

Data Analysis and Interpretation: T To, W Soo, H Lane Statistical Analysis: T To

Manuscript Preparation: T To, W Soo, H Lane, A Khattak

Manuscript Editing: T To, W Soo, H Lane, A Khattak, B Devitt, H Dhillon, A Booms, J Phillips Manuscript Review: T To, W Soo, H Lane, A Khattak, B Devitt, H Dhillon, A Booms, J Phillips 


\section{References}

1. Soto-Perez-de-Celis E, de Glas NA, Hsu T, Kanesvaran R, Steer C, Navarrete-Reyes AP, et al. Global geriatric oncology: Achievements and challenges. Journal of Geriatric Oncology 2017;8(5):374-86. doi: 10.1016/j.jgo.2017.06.001

2. Australian Government Department of Health. Geriatric medicine 2016 Factsheet. Canberra, Australia. 2016.

3. McCleary NJ, Wigler D, Berry D, Sato K, Abrams T, Chan J, et al. Feasibility of computer-based selfadministered cancer-specific geriatric assessment in older patients with gastrointestinal malignancy. The oncologist 2013;18(1):64-72. doi: 10.1634/theoncologist.2012-0241 [published Online First: 2013/01/05]

4. Jonker JM, Smorenburg CH, Schiphorst AH, van Rixtel B, Portielje JE, Hamaker ME. Geriatric oncology in the Netherlands: a survey of medical oncology specialists and oncology nursing specialists. Eur J Cancer Care (Engl) 2014;23(6):803-10. doi: 10.1111/ecc.12193

5. Pang A, Ho S, Lee SC. Cancer physicians' attitude towards treatment of the elderly cancer patient in a developed Asian country. BMC Geriatr 2013;13:35. doi: 10.1186/1471-2318-13-35

6. Honecker F, Wedding U, Huschens S, Bokemeyer C. Incorporation of geriatric assessment into oncology practive: Views and attitudes of physicians participating in the IN-GHO registry. $J$ Clin Oncol 2011;29(15_suppl):e19607-e07. doi: doi:10.1200/jco.2011.29.15_suppl.e19607

7. Wan-Chow-Wah D, Monette J, Monette M, Sourial N, Retornaz F, Batist G, et al. Difficulties in decision making regarding chemotherapy for older cancer patients: A census of cancer physicians. Critical reviews in oncology/hematology 2011;78(1):45-58. doi: 10.1016/j.critrevonc.2010.02.010 [published Online First: 2010/03/26]

8. Extermann M, Boler I, Reich RR, Lyman GH, Brown RH, DeFelice J, et al. Predicting the risk of chemotherapy toxicity in older patients: the Chemotherapy Risk Assessment Scale for HighAge Patients (CRASH) score. Cancer 2012;118(13):3377-86. doi: 10.1002/cncr.26646 [published Online First: 2011/11/11]

9. Hurria A, Togawa K, Mohile SG, Owusu C, Klepin HD, Gross CP, et al. Predicting chemotherapy toxicity in older adults with cancer: a prospective multicenter study. Journal of clinical oncology : official journal of the American Society of Clinical Oncology 2011;29(25):3457-65. doi: 10.1200/JCO.2011.34.7625 [published Online First: 2011/08/04]

10. Kenis C, Heeren P, Decoster L, Van Puyvelde K, Conings G, Cornelis F, et al. A Belgian Survey on Geriatric Assessment in Oncology Focusing on Large-Scale Implementation and Related Barriers and Facilitators. The journal of nutrition, health \& aging 2016;20(1):60-70. doi: 10.1007/s12603-015-0538-4 [published Online First: 2016/01/06]

11. Caillet P, Canoui-Poitrine F, Vouriot J, Berle M, Reinald N, Krypciak S, et al. Comprehensive geriatric assessment in the decision-making process in elderly patients with cancer: ELCAPA study. Journal of clinical oncology : official journal of the American Society of Clinical Oncology 2011;29(27):3636-42. doi: 10.1200/jco.2010.31.0664 [published Online First: 2011/06/29]

12. Chaibi P, Magne N, Breton S, Chebib A, Watson S, Duron JJ, et al. Influence of geriatric consultation with comprehensive geriatric assessment on final therapeutic decision in elderly cancer patients. Critical reviews in oncology/hematology 2011;79(3):302-7. doi: 10.1016/j.critrevonc.2010.08.004 [published Online First: 2010/10/05] 